Article

\title{
Peracetic Acid Sterilization Induces Divergent Biological Response in Polymeric Tissue Engineering Scaffolds
}

\author{
Suyog Yoganarasimha ${ }^{1}$, Al Best ${ }^{2}$ and Parthasarathy A. Madurantakam ${ }^{3,4, *}$ \\ 1 Department of Biomedical Engineering, School of Engineering, Virginia Commonwealth University, \\ Richmond, VA 23284, USA \\ 2 Departments of Biostatistics and Periodontics, School of Dentistry, Virginia Commonwealth University, \\ Richmond, VA 23298, USA \\ 3 Philips Institute of Oral Health Research, School of Dentistry, Virginia Commonwealth University, \\ Richmond, VA 23298, USA \\ 4 Department of General Practice, School of Dentistry, Virginia Commonwealth University, Richmond, \\ VA 23298, USA \\ * Correspondence: madurantakap@vcu.edu; Tel.: +1-804-828-4953
}

Received: 3 July 2019; Accepted: 30 August 2019; Published: 5 September 2019

check for updates

\begin{abstract}
Synthetic polymers offer control over composition, architecture, mechanical properties and degradation kinetics. Predictable sterilization of synthetic polymeric scaffolds made from low temperature melting polymers, remains a challenge to clinical translation. We previously demonstrated successful room temperature sterilization of electrospun polycaprolactone scaffolds (ePCL) using peracetic acid (PA). The current paper investigates the effects of PA sterilization on two different scaffolds types-ePCL and commercially available porous polystyrene (Alvetex ${ }^{\circledR}$ ) scaffolds using mouse calvarial osteoblasts cell line (MC3T3) and Live-Dead Assay. We report cytotoxicity in PA-treated ePCL scaffolds (PA-ePCL), while control scaffolds strongly supported cell survival. Treatment of PA-ePCL scaffolds with known methods of PA residual elimination (sodium thiosulfate, catalase, washing and aeration) had minimal effect on MC3T3 survival. However, incubation in $80 \%$ ethanol for 30 min successfully eliminated the toxic PA residuals and restored scaffold cytocompatibility. On the other hand, PA treatment of Alvetex ${ }^{\circledR}$ scaffolds induced diametrically opposite effects: cell survival and proliferation was enhanced after PA exposure and these responses were reversed following ethanol wash. These results suggest that PA treatment can induce different biological effects based on polymer chemistry and scaffold architecture and presents interesting opportunities to modulate biological properties of tissue engineering scaffolds.
\end{abstract}

Keywords: peracetic acid; sterilization; electrospinning; polymeric scaffolds; cytotoxicity; polycaprolactone; polystyrene; quenching; Alvetex

\section{Introduction}

Organ failure due to infection, injury or disease is a major health care issue that places enormous burden on the national economy. Restoration of structure and function of these tissues is essential for maintenance of quality of life. Current strategies include transplantation of grafts, mechanical devices and artificial prostheses. However, neither of these options result in satisfactory long-term outcomes. Tissue engineering is a promising strategy that attempts to create functional tissues by implanting synthetic or natural biomaterials that eventually are replaced by host tissue [1].

Strategies promoting tissue regeneration include cell-based approaches (involving transplantation of autologous/allogeneic cells [2-4], use of biological molecules including growth factors, DNA 
and polysaccharides [5-8], as well as scaffold-based tissue engineering approaches [9-13]. Currently, complex 3D tissue constructs are being engineered by culturing specific cell types in microenvironments that mimic the biochemical and mechanical aspects of natural extracellular matrix. Foremost among the list of requirements of implanted tissue engineered scaffold is its cytocompatibility. Beyond this basic requirement, the scaffolds need to be conducive to cell attachment, migration, proliferation, differentiation and maintenance of cell phenotype [14-17].

Synthetic resorbable polyesters are a popular choice in scaffold fabrication because they offer control over mechanical properties, degradation rates and micro/nano architecture [18,19]. Polycaprolactone (PCL) is relatively inexpensive aliphatic polyester with good solubility and exceptional blend-compatibility that lends itself to a variety of fabrication methods. Electrospinning is a versatile scaffold fabrication technique that consistently reproduces the submicron diameter fibers from polymer solutions. In addition to rendering the morphology similar to native ECM (extracellular matrix) the process is readily scalable; allows control over fiber diameter [20,21], porosity [22-24] and alignment $[25,26]$.

While most in vitro and in vivo studies using electrospun PCL have used ethanol disinfection prior to cell culture or implantation in animals, clearly this is inadequate for clinical translation for tissue-engineered product. Our previous study found that conventional methods of sterilization (autoclave, EtO) of low melting electrospun PCL scaffolds resulted in loss of fibrous architecture whereas peracetic acid (PA) at 1000 ppm preserved scaffold morphology [27].

PA is available as an equilibrium mixture of acetic acid and hydrogen peroxide and has been extensively used in the food industry because of its high potency and low residual toxicity. PA is effective at low concentrations, low temperatures and degrades into nontoxic end products (water, oxygen and carbon dioxide) and can be safely disposed of without affecting the environment. PA is among the list of Food and Drug Administration (FDA)-cleared sterilants cleared by the FDA for the processing of reusable medical and dental devices [28].

Unlike physical methods of sterilization (radiation and heat), an inherent issue using a chemical sterilant (liquid or gas) is the adsorbed residuals that need to be completely eliminated. Ethylene oxide (EtO), an effective chemical sterilant, is known for its adsorption onto polymeric surfaces and leave residuals that are both cytotoxic and carcinogenic. Extensive aeration (up to $12 \mathrm{~h}$ ) at $50-60{ }^{\circ} \mathrm{C}$ is necessary to completely remove all EtO residues [29] and is a part of standard operating procedures. Having introduced PA previously, we focused on evaluating its adsorption potential and cytotoxic effects on electrospun PCL (ePCL) scaffolds. To the best of our knowledge, this is the first study to evaluate the biological effects of PA sterilization on ePCL scaffolds and compare it against a commercially available polystyrene scaffold (Alvetex ${ }^{\mathrm{TM}}$ ). We further present appropriate protocols for elimination of PA residues to restore scaffold cytocompatibility.

\section{Materials and Methods}

\subsection{Electrospinning PCL Scaffolds}

PCL (Sigma, MW 80,000, Saint Louis, MO, USA) was dissolved in a binary solvent system of 1 formic acid: 3 acetic acid (FAA) at a concentration of $100 \mathrm{mg} / \mathrm{mL}$ [30]. Electrospinning apparatus (EC-DIG, IME Technologies, Van Dijklaan, The Netherlands) was used at optimized process conditions (rate: $7 \mathrm{~mL} / \mathrm{h}$, air-gap distance: $12.5 \mathrm{~cm}$, applied needle voltage: $+25 \mathrm{kV}$ ) to generate continuous, non-woven fibers that were collected onto $18 \mathrm{~mm}$ glass cover slips attached to cylindrical drum mandrel (100 mm diameter, IME Technologies, Van Dijklaan, The Netherlands) rotating at $100 \mathrm{rpm}$. After electrospinning, cover slips were removed from the mandrel, dried in a fume hood overnight and stored in an airtight desiccator until use. In order to test the potential differences in the solvent system, an additional set of PCL scaffolds was electrospun from hexafluro-2-isopropanol (HFP, Oakwood Products, Estill, SC, USA), according to our previous study [27]. 


\subsection{Scanning Electron Microscopy}

Air-dried electrospun scaffolds (before and after various sterilization protocols) were mounted on aluminum stubs using standard double-sided tape, sputter coated with gold and examined at an accelerating voltage of $20 \mathrm{kV}$ using JEOL JSM 5610LV (Peabody, MA, USA) scanning electron microscope (SEM). Average fiber diameters were calculated from a total of 50 randomly selected fibers from corresponding SEM images using Image J (NIH). For imaging cells seeded onto scaffolds, the scaffolds were fixed in $2.5 \%$ glutaraldehyde, sequentially dehydrated in ethanol, followed by $5 \mathrm{~min}$ incubation in gradations of hexamethyldisilazane (HMDS, Sigma, Saint Louis, MO, USA) prior to imaging. Alvetex scaffolds were also imaged under the same conditions.

\subsection{Scaffold Sterilization}

The scaffolds on coverslips were sterilized using previously identified conditions for peracetic acid (PA) sterilization. Stock PA (390,000 ppm) was diluted in 20\% ethanol to $1000 \mathrm{ppm}$ and ePCL were incubated for $15 \mathrm{~min}$ at room temperature. This group will be referred to as PA-ePCL. Control scaffolds were treated with $70 \%$ ethanol for $30 \mathrm{~min}$ and referred to as Ctrl-ePCL. All scaffolds were washed thrice with $30 \mathrm{~mL}$ of DI water (10 min each) before cell seeding.

\subsection{Cytotoxicity Assay}

For the experiments listed in Sections 2.5-2.7 below, we used Live/Dead assay (Life Technologies) at $24 \mathrm{~h}$ after cell seeding to screen the scaffolds for cytotoxicity. A total of 20,000 MC3T3 cells were seeded at the center of scaffold and retained within a sterile $10 \mathrm{~mm}$ diameter, glass-cloning ring (Corning). Cells were cultured in DMEM (Dulbecco Modified Eagle Media) with 10\% FBS (Atlanta Biologicals, Flowery Branch, GA, USA) and 1\% antibiotic/antimycotic (Invitrogen) at $37^{\circ} \mathrm{C}$ and $5 \%$ $\mathrm{CO}_{2}$ for $24 \mathrm{~h}$. The assay was performed according to manufacturer's instructions and scaffolds were imaged at 5 different fields using Nikon fluorescence microscope. Live cells with esterase activity will emit green fluorescence while dead cells with exposed DNA emit red fluorescence. Representative images are presented as an overlay of green and red channels at 10× magnification for all experiments. Cells cultured on tissue culture polystyrene (TCPS) were used as positive controls.

\subsection{Chemical Neutralization and Physical Decontamination}

PA is a powerful oxidizer and is available in an equilibrium mixture of peracetic acid (39\%), hydrogen peroxide $(6 \%)$ in acetic acid. We investigated PA neutralization using $2 \%$ sodium thiosulfate (STS, Sigma, Saint Louis, MO, USA), a known reducing agent [31] and hydrogen peroxide quenching using catalase $(0.1 \mathrm{mg} / \mathrm{mL})$. Further, we also adapted the aeration protocol currently used to eliminate residuals following EtO sterilization. After chemical neutralization with STS and catalase, we placed PA-treated and control ePCL scaffolds in a custom chamber and purged with air for $18 \mathrm{~h}$ to effect decontamination and elimination of PA residues. After aeration, the scaffolds were washed in DI water ( $3 \times$ for $10 \mathrm{~min}$ each) prior to cell seeding.

\subsection{PA Quenching Using Ethanol}

Since ethanol is used to improve wetting characteristics of hydrophobic scaffolds and has molecular size similar to PA, we hypothesized that ethanol could effectively desorb PA from electrospun scaffolds. Accordingly, we quenched PA-scaffolds in $80 \%$ ethanol for $30 \mathrm{~min}$ followed by washing thrice in DI water (10 min each), prior to cell seeding.

\subsection{Cell Proliferation Assay}

Having observed the reversal of cytotoxicity in PA-ePCL after ethanol quenching, we evaluated if these biological effects were stable over time. In order to verify if PA-induced cytotoxicity was specific to ePCL, we chose to study commercially available Alvetex scaffold (Reinnervate Ltd. 
Durham, UK). The rationale behind this choice was that it was made from polystyrene; the scaffolds have macropores and they do not have nanofibrous architecture. Four groups were accordingly identified-electrospun PCL treated with PA (PA-ePCL), PA-treated electrospun PCL quenched with ethanol (PA-ePCL+EtOH), Alvetex scaffolds treated with PA (PA-Alv) and PA-treated Alvetex quenched with ethanol (PA-Alv+EtOH). Control scaffolds, disinfected with $70 \%$ ethanol for $30 \mathrm{~min}$, were designated Ctrl-ePCL and Ctrl-Alv. All scaffolds were washed thrice in PBS for 10 min each prior to cell seeding. MC3T3 cells were seeded at a density of 10,000 cells $/ \mathrm{cm}^{2}$ and a modified MTS assay (Promega, Madison, WI, USA) performed on days 1 and 7 [32]. Briefly, cell-seeded scaffolds were washed with PBS at designated time points and incubated with MTS reagents for $2 \mathrm{~h}$. The scaffolds were then boiled in 1\% Triton X-100 lysis buffer to ensure MTS product was removed from the scaffolds and cells. The supernatant was then read for absorbance at $490 \mathrm{~nm}$ on a BioTek Synergy 2 microplate reader.

\subsection{Statistical Analyses}

Experiments were performed in triplicate and repeated twice to improve validity. Optical density (OD) measurements were analyzed using a mixed-model, repeated-measures ANOVA. Since OD was skewed, it was analyzed on the log scale and then the results back transformed to the original units and expressed as ratio of absorbance at day7:day1. SAS software was used for all analyses (SAS Institute, Inc., Cary NC, USA).

\section{Results}

\subsection{Scaffold Generation}

The scaffolds (75-100 $\mu \mathrm{m}$ thick) generated from both solvent systems (FAA (Formic acid: Acetic acid) and HFP (Hexafluroisopropanol)) yielded fibrous scaffolds; however, the type of solvent had the greatest impact on the dimension of the fibers. While PCL electrospun from HFP showed a typical bimodal distribution of fiber diameters (average $1.37 \mu \mathrm{m}$ ), fibers electrospun from FAA were on average 6 times smaller $(0.21 \mu \mathrm{m})$ and also had more uniform fiber size distribution (Figure 1).
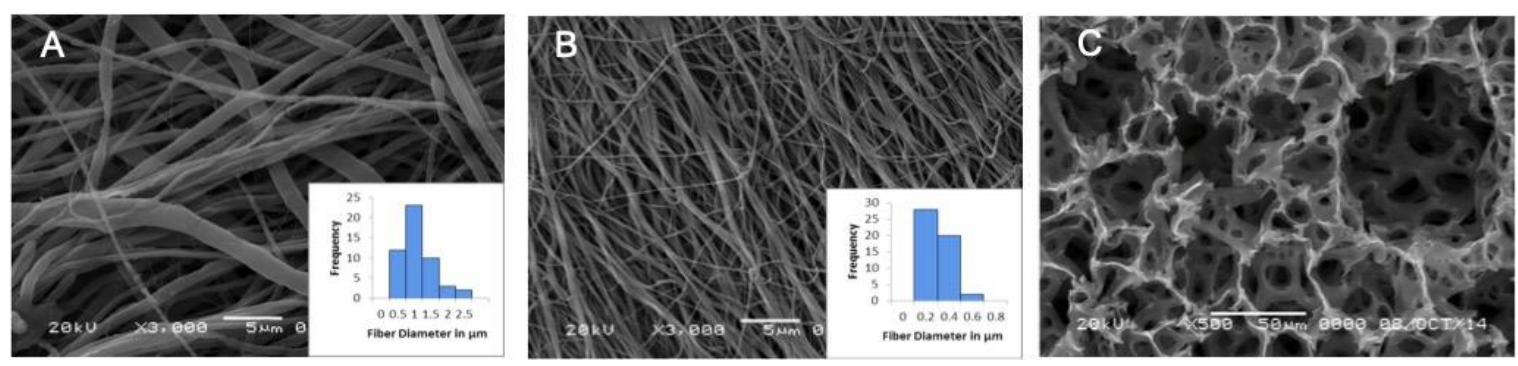

Figure 1. PCL scaffolds electrospun from (A) HFP and (B) a binary solvent system of formic acid and acetic acid (1:3). PCL fibers from HFP show a bimodal distribution with mean diameter of $1.37 \mu \mathrm{m}$. PCL electrospun from FAA show a narrow fibers (mean diameter of $0.21 \mu \mathrm{m}$ ) but much more uniform fiber size distribution. Panel $(\mathbf{C})$ is the image of macroporous Alvetex scaffolds demonstrating interconnected pore structure.

\subsection{PA Sterilization on Cellular Viability}

Since PA sterilization does not induce any physical change in ePCL, we expected no significant effects on their cytocompatibility. However, the viability of MC3T3 showed completely different profiles when cultured on Ctrl-ePCL scaffolds and PA-PCL after $24 \mathrm{~h}$. Live/dead staining (Figure 2) revealed robust cell attachment and survival on Ctrl-ePCL while PA-ePCL was completely cytotoxic. There was a significantly smaller number of cells on PA-ePCL and all were dead within $24 \mathrm{~h}$.

It is also interesting to notice the effects of PA on ePCL scaffolds electrospun from different solvents- HFP and FAA (Figure 3). It can be seen that PA treatment of ePCL generated from HFP was 
less cytotoxic compared to those fabricated from FAA. Even though greater number of cells survived on HFP generated scaffolds, they showed altered morphology. These results indicated that in spite of having the same polymer, the adsorption profile of PA and subsequent biologic response differed based on the solvent used for electrospinning. Fourier transform infrared (FTIR) analysis failed to detect any difference between the scaffold types (data not shown). Since ePCL generated from FAA had favorable scaffold morphology and was associated with significant cytotoxicity following PA treatment, we focused all subsequent experiments on these scaffolds.
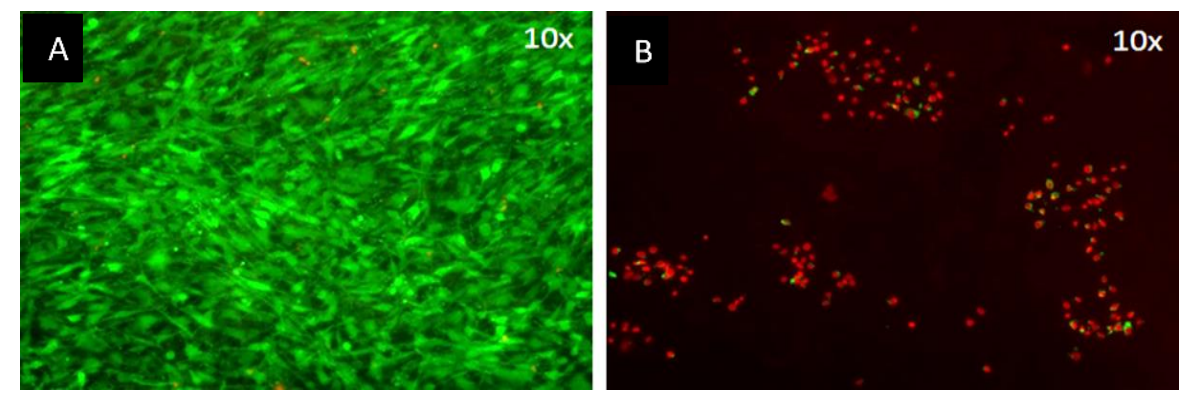

Figure 2. Effect of PAA on cell viability. Live/Dead staining of MC3T3 on Ctrl PCL scaffold with no PAA exposure (A) show robust cell attachment while PAA sterilized PCL scaffold (B) is profoundly cytotoxic.

Ctrl- PCL scaffold
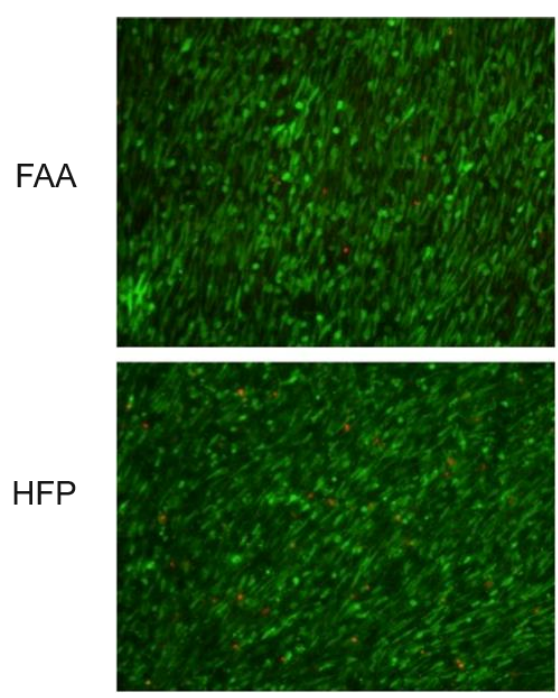

PA- ePCL scaffold
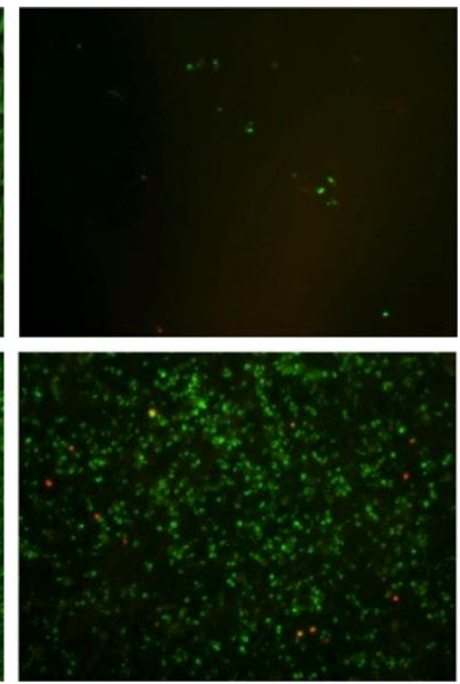

Figure 3. Effect of PAA on electrospun PCL scaffolds generated from two solvent systems. The top panel is PCL electrospun from formic acid: acetic acid while the bottom panel is from HFP. While control scaffolds (with no PAA exposure) show identical cell attachment, exposure of PAA induces a markedly different response. Whereas PAA-ES from formic acid: acetic acid is completely cytotoxic, scaffolds from HFP show moderate cell survival although with altered cell morphology. All images are at 10X magnification.

\subsection{Chemical Neutralization and Physical Decontamination}

As can be seen from Figure 4, neither STS (Sodium thiosulfate) nor catalase was cytotoxic at the concentrations used in the experiments. Incubation of PA-ePCL with STS did not improve the cytocompatibility profile while catalase treatment indicated a weak potential to support cell survival. There was an increase in the number of attached cells as well as a higher proportion of live cells. These results show that STS was not adequate to neutralize or eliminate the adsorbed PA residuals while catalase was mildly effective. PA-ePCL, aerated for $18 \mathrm{~h}$, showed a remarkable decrease in the number of dead cells with a concomitant increase in living cells even though the cell density is 
significantly less than the controls. This result suggests that aeration was moderately effective in improving scaffold cytocompatibility.

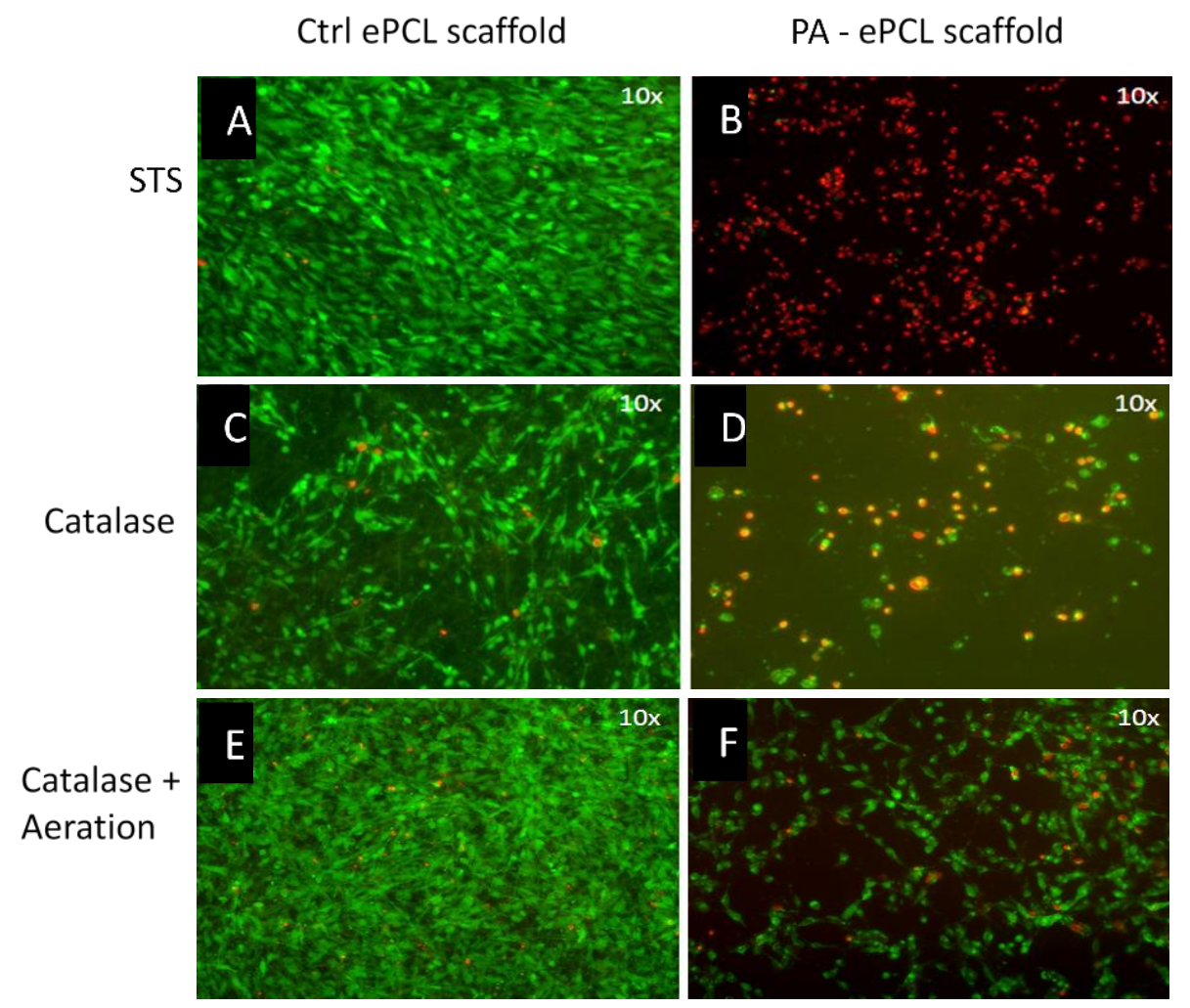

Figure 4. Effect of PA neutralization by STS (top panel); catalase (middle panel); catalase and aeration (bottom panel) on 24-h viability of MC3T3. Panels (A), (C) and (E) refer to control ePCL scaffolds disinfected with $70 \%$ ethanol while (B), (D) and (F) are PA-treated. As can be seen, STS had no effect on cell viability; catalase was able to partially recover cell survival. Catalase combined with extensive aeration was more effective in overcoming the cytotoxic effects of PA treatment. It is important to note that control ePCL scaffolds exposed to STS, catalase and aeration protocols showed robust cell survival (left panel).

\subsection{PA Quenching with Ethanol}

The effect of quenching PA-treated scaffolds in $80 \%$ ethanol for $30 \mathrm{~min}$ is illustrated in Figure 5. As can be seen, ethanol treatment fully restored the scaffold cytocompatibility to levels comparable to controls. The cell density, flattened cell morphology and the proportion of live cells were similar to that of ethanol disinfected scaffolds. Scanning electron microscopy demonstrated similar cell spreading behavior on ethanol quenched PA-ePCL compared to controls (Figure 6).
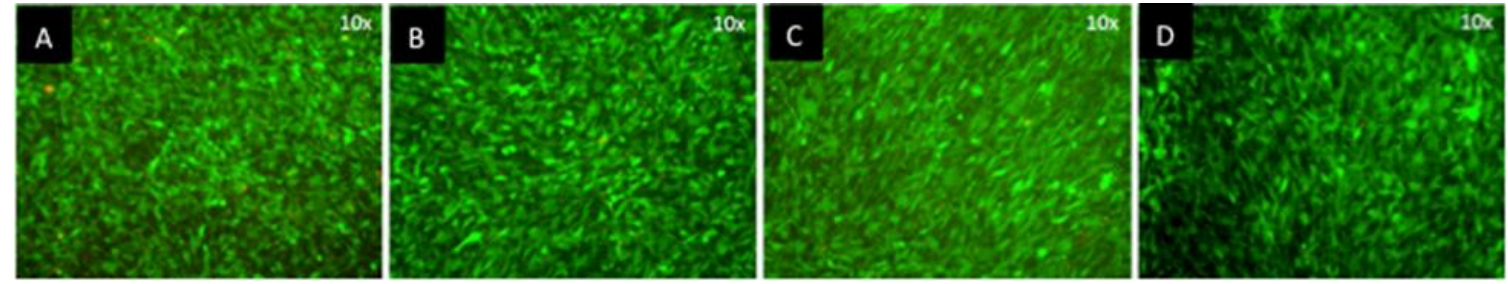

Figure 5. Cell viability is restored in PA-treated ePCL scaffolds by ethanol wash and is not affected by concentration of PAA. Live/Dead staining of MC3T3 on scaffolds with no exposure to PAA (A), 1000 ppm (B), 2500 ppm (C) and 5000 ppm (D). It can be readily seen that the cell attachment and survival in PAA treated scaffolds is comparable to controls. 

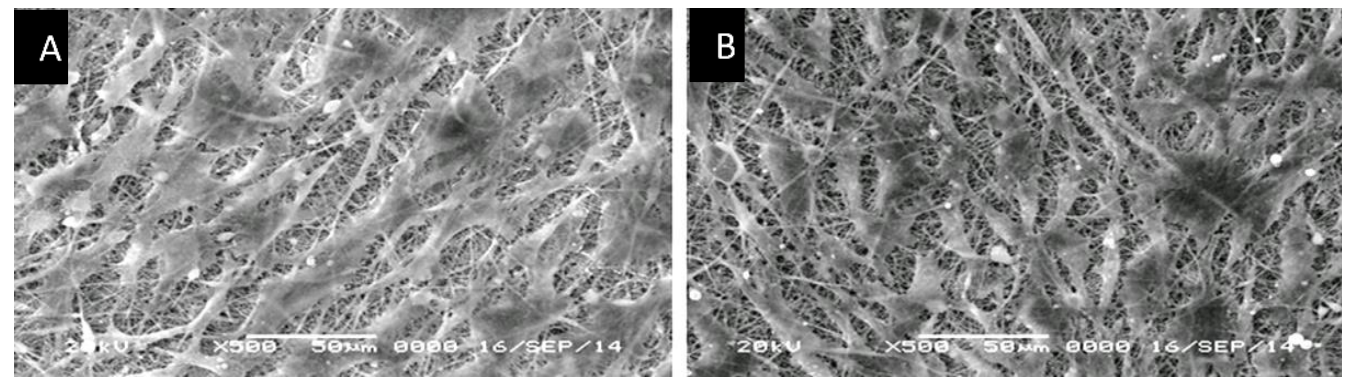

Figure 6. Scanning electron microscopy (SEM) images of MC3T3 after 24-h culture on Ctrl ePCL (A) and PA-ePCL treated with ethanol (B) show similar density and spreading behavior. This illustrates successful removal of PA residuals from ePCL by ethanol wash.

\subsection{Cell Proliferation (MTS) Assay}

We established the MTS standard curve using different numbers of MC3T3 $\left(R^{2}=0.9981\right.$, data not shown) to validate the assay protocol. The most obvious inference from Figures 7 and 8 is that there is a significant increase in cell numbers from day 1 to day 7 in all groups with the exception of PA-ePCL group. This is consistent with the previous observation from Live/Dead assay and confirms the cytotoxicity of PA treatment. Importantly, there is no statistically significant difference in the day 1 cell viability between Ctrl-ePCL and PA-ePCL+EtOH groups $(p=0.9079)$. This indicates the effectiveness of ethanol quench in overcoming the negative effects of PA. We further notice that despite having similar number of cells at day 1, Ctrl-ePCL and PA-ePCL+EtOH were significantly different in their ability to support cell proliferation at day $7(p=0.013)$. In both cases, the increase in cell numbers was moderate (ranging from 25-60\%).

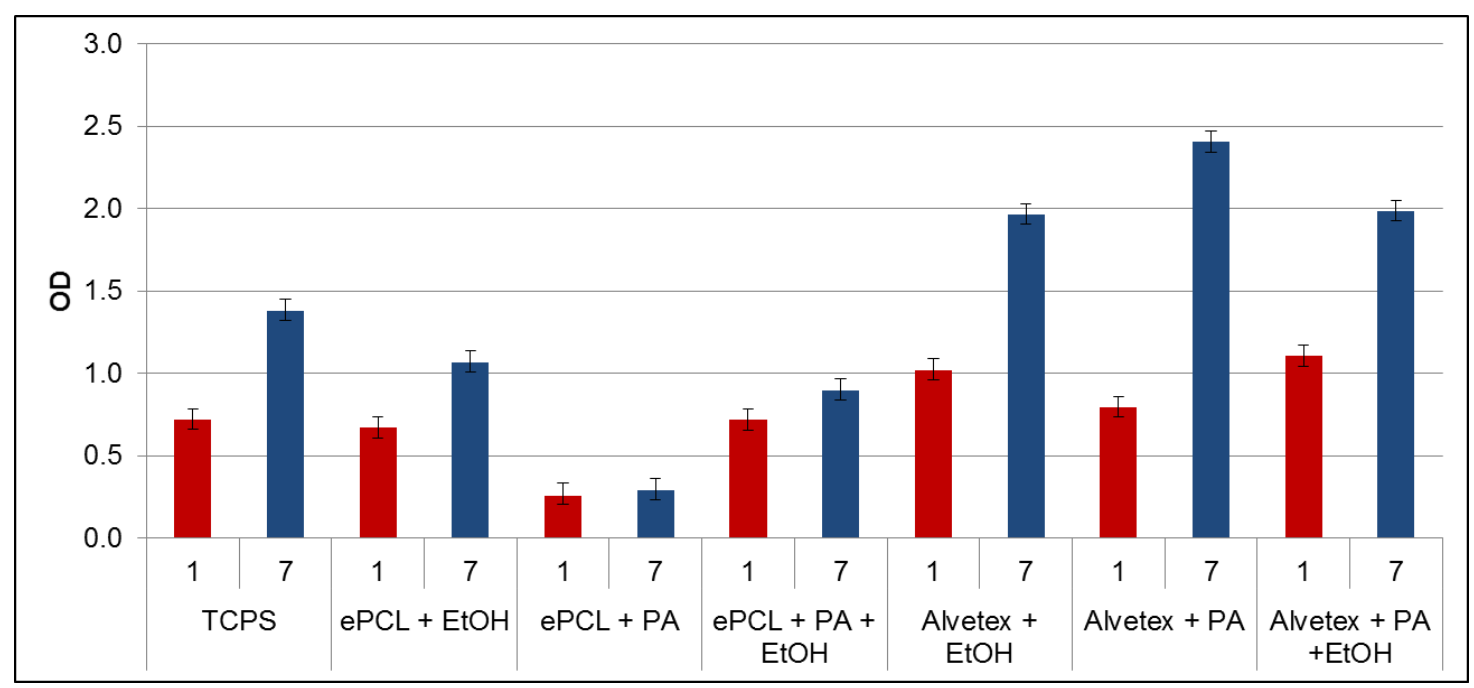

Figure 7. Cell proliferation data from MTS assay. Raw OD from 1-day and 7-day culture of MC3T3 osteoblasts across different substrates. All substrates show positive cell proliferation from day-1 to day-7 with the exception of ePCL treated with PA. There was no detectable signal at day- 1 and day 7 indicating complete cytotoxicity of these scaffolds following PA exposure. Alvetex on the other hand showed increased cell proliferation after PA exposure. PA effects were effectively neutralized following ethanol wash. 


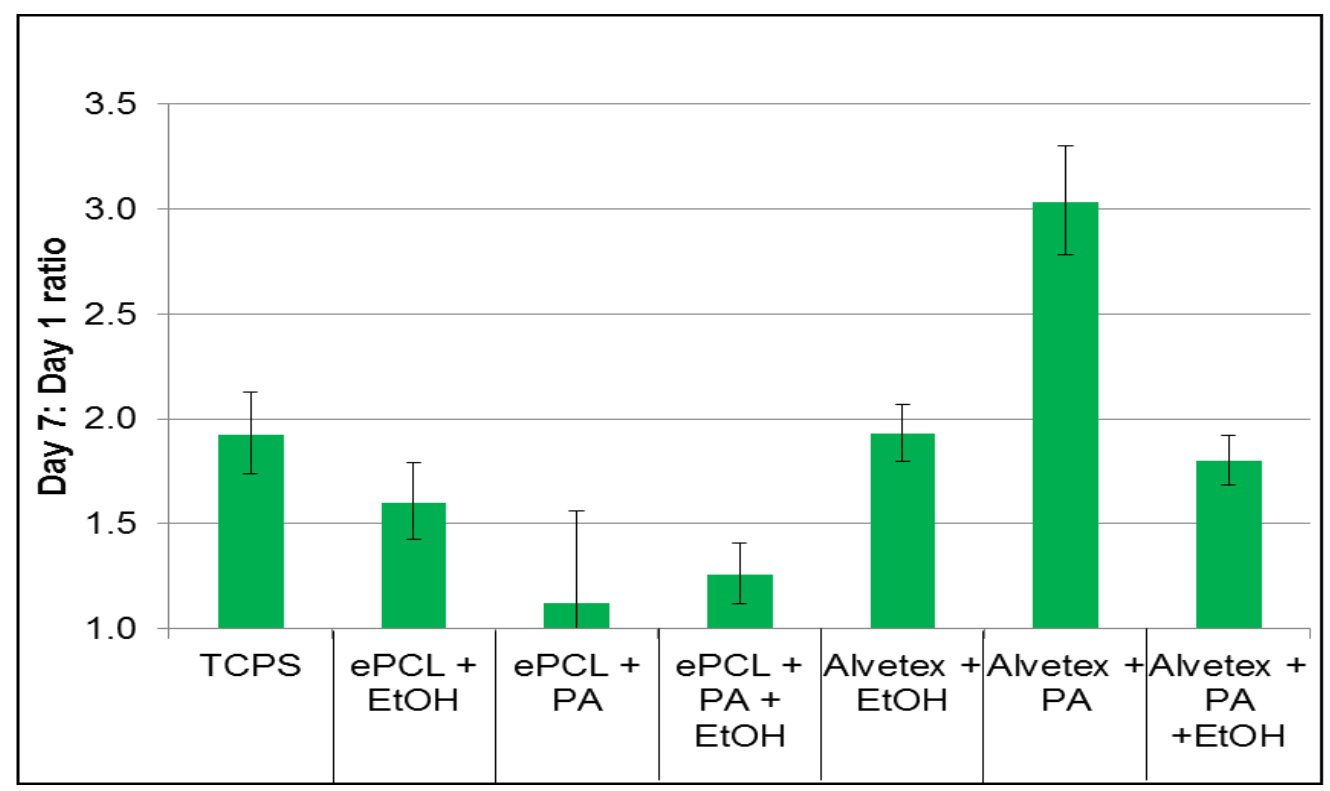

Figure 8. Differential effects of PAA on various substrates. Data is presented as a ratio of optical densities from day 7 to day 1 to account for significant differences in initial (day 1 ) cell attachment on different surfaces. Since each scaffold serves as its internal control, this approach allows comparison of cell proliferation across scaffolds. While ES+PA+ EtOH supported early cell survival and limited cell proliferation, it was significantly lower than Ctrl ES. PA+ Alvetex showed maximal cell proliferation, which decreased following ethanol wash.

In complete contrast to ePCL, Alvetex scaffolds were not cytotoxic after PA treatment at day 1. This reduced adsorption of PA components can be a result of different material chemistry (PCL vs. polystyrene), scaffold architecture and specific surface area (nanofibrous PCL vs. macroporous Alvetex). In addition, cells seeded on Alvetex demonstrated robust cell proliferation that was significantly higher than ePCL (100-200\%). Interestingly, PA-Alv showed maximum proliferation and if PA-Alv were washed with ethanol, the proliferation rates return back to baseline (Ctrl-Alv). This again indicates that ethanol was effective in reversing the PA-induced change in Alvetex polystyrene scaffolds.

\section{Discussion}

In addition to being non-toxic, tissue-engineering scaffolds need to be biologically active; integrate with the tissues, undergo remodeling and be eventually replaced by the host tissue. In order for these events to occur, it is imperative that the scaffolds act as a favorable substrate that support appropriate cell activity, including attachment, migration and proliferation through the thickness of the scaffold. PCL is aliphatic polyester, readily soluble in a variety of solvents used in manufacturing. Further, being a synthetic polymer, the degradation rates and mechanical properties can be readily controlled. The fact that a number of drug-delivery devices fabricated with PCL already have FDA approval makes this polymer really attractive for tissue engineering purposes [33].

Irrespective of its biomaterial, fabrication technique or its intended clinical application any implanted biomaterial must be sterile prior to implantation. Even though a variety of physical (autoclaving, dry heat and irradiation) or chemical (ethylene oxide gas or peracetic acid liquid) methods exist, our lab has reported conventional sterilization techniques (including EtO) induced PCL scaffold degradation, while peracetic acid treatment (1000 ppm for $15 \mathrm{~min}$ at room temperature) effectively sterilized the scaffolds without adversely affecting its properties.

Since PA is an equilibrium mixture of acetic acid, hydrogen peroxide and water, presence of toxic residuals within the biomaterial can contribute to cytotoxicity and hence remains a concern. Given the efficacy of PA in sterilizing polymeric scaffolds at room temperature, the focus of this study was 
to address the issue of cytotoxicity after PA treatment. Our cytotoxicity results in ePCL is consistent with the findings of Franklin et al. [34], who reported significant cytotoxicity associated with low temperature hydrogen peroxide gas plasma treatment of PCL scaffolds. The authors suggested that the leaching of residual hydrogen peroxide from within or upon the porous scaffolds could explain observed cytotoxic effects. Other studies have reported adsorption of known chemical and gas sterilants (hydrogen peroxide and $\mathrm{EtO}$ ) onto polymers with some polymer absorbing considerable amounts of $\mathrm{H}_{2} \mathrm{O}_{2}$ in less than an hour. [32,35] Studies exist in the literature regarding use of STS and catalase to remove residual concentrations of PA. [36]. Radl et al. demonstrated that desorption kinetics from polymers exposed to hydrogen peroxide showed a strong dependence on the material composition and temperature [29]. These studies formed the basis for the physico-chemical methods we employed to desorb PA residues from electrospun scaffolds in this study.

Since conventional processing of scaffolds prior to experiments is to treat them with ethanol followed by 3 washes with DI/ PBS 10 min per wash and the solutions being changed for every wash, we started off with this strategy. Extensive washing (irrespective of volume or the number of times solutions were changed) or total incubation time (up to 2 days) were any more effective than washing for $30 \mathrm{~min}$. Looking at the minimal effect of washing on removing PA residuals, we turned our attention to using published physical and chemical methods of neutralizing PA residuals using STS, catalase and aeration. With limited success, we chose to investigate the effect of ethanol wash on PA-ePCL scaffolds. Support for this strategy came from few studies that reported use of peracetic acid-ethanol solution for sterilizing bone allografts $[37,38]$. Even though peracetic acid itself was sufficient for achieving sterilization, additional steps-application of negative pressure (200 mbar); addition of ethanol (approximately 24\%) and gentle agitation were required to ensure predictable sterilization throughout the thickness of the allografts. We hypothesized that ethanol and peracetic acid being similar sized molecules and with ethanol possessing better wetting capabilities, PA-treatment followed by ethanol wash may result in effective desorption of PA residues. We confirmed the effectiveness of ethanol wash in additional experiments on solid, non-porous, poly-ether-ether ketone (PEEK) surfaces and MG-63 cells (data not shown).

While observing cytotoxicity in PA-ePCL scaffolds, we recognized that effect of PA cannot be generalized and may depend on substrate chemical composition and architecture. The evidence came from a recently published study, which reported no cytotoxicity following PA treatment of decellularized cadaveric tendons. [39] While differences in terms of available surface area and post-PA alkali neutralization could have accounted for different observations, it provided an impetus to look into an alternate scaffold to compare. The decision to choose Alvetex was because it was commercially available, made from polystyrene, generated by high internal phase emulsion and has porosity $>90 \%$ with regular ( 40um) interconnected pores [40].

We reasoned direct comparison of commercially available Alvetex and in-house generated ePCL would allow identify if the effect of PA would be affected by scaffold composition, geometry and architecture. While Alvetex was superior to ePCL in terms of cell proliferation both short and long term, the more interesting finding was the improved performance of Alvetex scaffolds that were treated with PA indicating some favorable surface changes induced on polystyrene surfaces. The mechanisms underlying these changes are currently being investigated. Contact angle measurements done on Alvetex scaffolds following different treatments revealed no significant differences between conventional ethanol treatment (manufacturer recommendation) and PA treatment (data not shown). These observations suggest that parameters other than scaffold hydrophilicity are responsible for the favorable cell response.

Among the limitations of the current study are use of two time points to assess cell proliferation. In the context of tissue engineering, a longer time point would prove beneficial. This would be possible with the use of bioreactors. We used scanning electron micrographs to provide a visual representation of cell-scaffold interactions at microscopic level. However, use of more sophisticated 
confocal microscopy and molecular markers of adhesion and spreading (f-Actin, vinculin, integrins etc.) would provide more insights into cell behavior.

Overall, the data from the current study has demonstrated that peracetic acid can be a viable sterilant for polymeric tissue engineering scaffold especially after verifying if the cytotoxic adsorbed residuals are removed. PA poses an interesting challenge because its adsorption profile was different in ePCL and polystyrene. Possible explanations could be differences in polymer chemistry, fabrication technique (including solvents used), scaffold architecture (porosity and available surface area) as well as additional post fabrication steps. Furthermore, by mechanisms that are not clear, PA treatment also positively influences cell proliferation in Alvetex. Importantly, we have developed a reliable strategy to reverse the effects of PA treatment using ethanol.

One of the important issues to consider in developing polymeric scaffolds for clinical application is the effect of sterilization on degradation kinetics. Since most polymers undergo hydrolysis (enzymatic or non-enzymatic) upon implantation, when implanted, this needs to be investigated further. This is even more relevant with peracetic acid since acid mediated hydrolysis of polymer chains is known to occur. Elucidation of multiple effects of as well as mechanisms underlying changes of polymeric surfaces after PA treatment presents an interesting area for future research.

\section{Conclusions}

The goal of the current study was to evaluate the cytocompatibility of electrospun polymeric scaffolds sterilized by PA using Live-Dead assay and MC3T3 cells. Live/Dead staining indicated massive cell death on PA treated ePCL scaffolds. These cytotoxic effects persisted after employing known methods to remove residuals including PBS washing, use of neutralizing agents (catalase and sodium thiosulfate) as well as extensive aeration. Washing PA treated scaffolds with $80 \%$ ethanol for $30 \mathrm{~min}$ resulted in recovery of cytocompatibility, compared to controls. However, the proliferation lagged behind the controls over a 7-day period. Alvetex scaffolds consistently performed better than ePCL following traditional ethanol disinfection. More interesting is the fact that there was significantly enhanced cell attachment and proliferation on Alvetex scaffolds after PA treatment. These data illustrates the differential effects of PA on various polymeric surfaces and provides interesting avenue to modulate cell behavior on tissue engineering scaffolds.

Author Contributions: For research articles with several authors, a short paragraph specifying their individual contributions must be provided. The following statements should be used "Conceptualization, P.M. and S.Y.; Methodology, P.M. and S.Y.; Formal analysis, A.B.; Writing—Original draft preparation, S.Y.; Writing—Review and editing, P.M.

Funding: The project was supported by CTSA (UL1TR000058) from the National Center for Advancing Translational Sciences) and the CCTR Endowment Fund of Virginia Commonwealth University. The contents are solely the responsibility of the authors and do not necessarily represent official views of the National Center for Advancing Translational Sciences or the National Institutes of Health.

Conflicts of Interest: The authors declare no conflict of interest.

\section{References}

1. Birla, R. Introduction to Tissue Engineering: Applications and Challenges; IEEE Press Series in Biomedical Engineering; John Wiley \& Sons Inc.: Hoboken, NJ, USA, 2014; pp. 2-5.

2. Matsuura, K.; Utoh, R.; Nagase, K.; Okano, T. Cell sheet approach for tissue engineering and regenerative medicine. J. Control Release 2014, 190, 228-239. [CrossRef] [PubMed]

3. Ma, J.; Both, S.K.; Yang, F.; Cui, F.Z.; Pan, J.; Meijer, G.J.; Jansen, J.A.; Jeroen, J.J.P.; van den Beucken, J.J. Concise review: Cell-based strategies in bone tissue engineering and regenerative medicine. Stem Cells Transl. Med. 2014, 3, 98-107. [CrossRef] [PubMed]

4. Bates, D.; Kampa, P. Cell-based regenerative approaches to the treatment of oral soft tissue defects. Int. J. Oral Maxillofac. Implants 2013, 28, e424-e431. [CrossRef] [PubMed]

5. Lee, K.; Silva, E.A.; Mooney, D.J. Growth factor delivery-based tissue engineering: General approaches and a review of recent developments. J. R. Soc. Interface 2011, 8, 153-170. [CrossRef] [PubMed] 
6. Chen, F.-M.; Zhang, M.; Wu, Z.-F. Toward delivery of multiple growth factors in tissue engineering. Biomaterials 2010, 31, 6279-6308. [CrossRef] [PubMed]

7. Gower, R.M.; Shea, L.D. Biomaterial Scaffolds for Controlled, Localized Gene Delivery of Regenerative Factors. Adv. Wound Care 2013, 2, 100-106. [CrossRef] [PubMed]

8. Aravamudhan, A.; Ramos, D.M.; Nip, J.; Subramanian, A.; James, R.; Harmon, M.D.; Kumbar, S.G. Osteoinductive small molecules: Growth factor alternatives for bone tissue engineering. Curr. Pharm. Des. 2013, 19, 3420-3428. [CrossRef] [PubMed]

9. Hutmacher, D.W.; Cool, S. Concepts of scaffold-based tissue engineering-the rationale to use solid free-form fabrication techniques. J. Cell. Mol. Med. 2007, 11, 654-669. [CrossRef] [PubMed]

10. Van Vlierberghe, S.; Dubruel, P.; Schacht, E. Biopolymer-based hydrogels as scaffolds for tissue engineering applications: A review. Biomacromolecules 2011, 12, 1387-1408. [CrossRef] [PubMed]

11. Wolf, M.T.; Dearth, C.L.; Sonnenberg, S.B.; Loboa, E.G.; Badylak, S.F. Naturally Derived and Synthetic Scaffolds for Skeletal Muscle Reconstruction. Adv. Drug Deliv. Rev. 2015, 84, 208-221. [CrossRef] [PubMed]

12. Shabafrooz, V.; Mozafari, M.; Vashaee, D.; Tayebi, L. Electrospun nanofibers: From filtration membranes to highly specialized tissue engineering scaffolds. J. Nanosci. Nanotechnol. 2014, 14, 522-534. [CrossRef] [PubMed]

13. Garg, T.; Goyal, A.K. Biomaterial-based scaffolds-Current status and future directions. Expert Opin. Drug Deliv. 2014, 11, 767-789. [CrossRef] [PubMed]

14. Chan, B.P.; Leong, K.W. Scaffolding in tissue engineering: G approaches and tissue-specific considerations. Eur. Spine J. 2008, 17 (Suppl. S4), 467-479. [CrossRef] [PubMed]

15. O’Brien, F.J. Biomaterials \& scaffolds for tissue engineering. Mater. Today 2011, 14, 88-95.

16. Boland, E.D.; Espy, P.G.; Bowlin, G.L. Tissue engineering scaffolds. In Encyclopedia of Biomaterials and Biomedical Engineering; CRC Press: Boca Raton, FL, USA, 2004; Volume 2, pp. 1630-1638.

17. Ma, Z.; Kotaki, M.; Inai, R.; Ramakrishna, S. Potential of nanofiber matrix as tissue-engineering scaffolds. Tissue Eng. 2005, 11, 101-109. [CrossRef] [PubMed]

18. Sachlos, E.; Czernuszka, J.T. Making tissue engineering scaffolds work. Review: The application of solid freeform fabrication technology to the production of tissue engineering scaffolds. Eur. Cell Mater. 2003, 5, 29-39. [CrossRef] [PubMed]

19. Yang, S.; Leong, K.F.; Du, Z.; Chua, C.K. The design of scaffolds for use in tissue engineering. Part I. Traditional factors. Tissue Eng. 2001, 7, 679-689. [CrossRef]

20. Badami, A.S.; Kreke, M.R.; Thompson, M.S.; Riffle, J.S.; Goldstein, A.S. Effect of fiber diameter on spreading, proliferation and differentiation of osteoblastic cells on electrospun poly (lactic acid) substrates. Biomaterials 2006, 27, 596-606. [CrossRef]

21. Balguid, A.; Mol, A.; van Marion, M.H.; Bank, R.A.; Bouten, C.V.; Baaijens, F.P. Tailoring fiber diameter in electrospun poly ( $\varepsilon$-caprolactone) scaffolds for optimal cellular infiltration in cardiovascular tissue engineering. Tissue Eng. Part A 2008, 15, 437-444. [CrossRef]

22. Baker, B.M.; Gee, A.O.; Metter, R.B.; Nathan, A.S.; Marklein, R.A.; Burdick, J.A.; Mauck, R.L. The potential to improve cell infiltration in composite fiber-aligned electrospun scaffolds by the selective removal of sacrificial fibers. Biomaterials 2008, 29, 2348-2358. [CrossRef]

23. Blakeney, B.A.; Tambralli, A.; Anderson, J.M.; Andukuri, A.; Lim, D.J.; Dean, D.R.; Jun, H.W. Cell infiltration and growth in a low density, uncompressed three-dimensional electrospun nanofibrous scaffold. Biomaterials 2011, 32, 1583-1590. [CrossRef] [PubMed]

24. Rnjak-Kovacina, J.; Weiss, A.S. Increasing the pore size of electrospun scaffolds. Tissue Eng. Part B Rev. 2011, 17, 365-372. [CrossRef] [PubMed]

25. Ayres, C.; Bowlin, G.L.; Henderson, S.C.; Taylor, L.; Shultz, J.; Alexander, J.; Telemeco, T.A.; Simpson, D.G. Modulation of anisotropy in electrospun tissue-engineering scaffolds: Analysis of fiber alignment by the fast Fourier transform. Biomaterials 2006, 27, 5524-5534. [CrossRef] [PubMed]

26. Chew, S.Y.; Mi, R.; Hoke, A.; Leong, K.W. The effect of the alignment of electrospun fibrous scaffolds on Schwann cell maturation. Biomaterials 2008, 29, 653-661. [CrossRef] [PubMed]

27. Yoganarasimha, S.; Trahan, W.R.; Best, A.M.; Bowlin, G.L.; Kitten, T.O.; Moon, P.C.; Madurantakam, P.A. Peracetic Acid: A Practical Agent for Sterilizing Heat-Labile Polymeric Tissue-Engineering Scaffolds. Tissue Eng. Part C Methods 2014, 20, 714-723. [CrossRef] [PubMed] 
28. FDA-Cleared Sterilants and High Level Disinfectants with General Claims for Processing Reusable Medical and Dental Devices-March 2015. Available online: https://www.fda.gov/medical-devices/reprocessingreusable-medical-devices-information-manufacturers/ (accessed on 8 June 2019).

29. Radl, S.; Larisegger, S.; Suzzi, D.; Khinast, J.G. Quantifying Absorption Effects during Hydrogen Peroxide Decontamination. J. Pharm. Innov. 2011, 6, 202. [CrossRef]

30. Van der Schueren, L.; De Schoenmaker, B.; Kalaoglu, Ö.I.; De Clerck, K. An alternative solvent system for the steady state electrospinning of polycaprolactone. Eur. Polym. J. 2011, 47, 1256-1263. [CrossRef]

31. Liu, W.; Andrews, S.A.; Stefan, M.I.; Bolton, J.R. Optimal methods for quenching $\mathrm{H}_{2} \mathrm{O}_{2}$ residuals prior to UFC testing. Water Res. 2003, 37, 3697-3703. [CrossRef]

32. Phipps, M.C.; Clem, W.C.; Catledge, S.A.; Xu, Y.; Hennessy, K.M.; Thomas, V.; Jablonsky, M.J.; Chowdhury, S.; Stanishevsky, A.V.; Bellis, S.L.; et al. Mesenchymal stem cell responses to bone-mimetic electrospun matrices composed of polycaprolactone, collagen I and nanoparticulate hydroxyapatite. PLoS ONE 2011, 6, e16813. [CrossRef]

33. Woodruff, M.A.; Hutmacher, D.W. The return of a forgotten polymer-Polycaprolactone in the 21st century. Prog. Polym. Sci. 2010, 35, 1217-1256. [CrossRef]

34. Franklin, S.P.; Stoker, A.M.; Cockrell, M.K.; Pfeiffer, F.M.; Bal, B.S.; Cook, J.L. Effects of Low-Temperature Hydrogen Peroxide Gas Plasma Sterilization on In Vitro Cytotoxicity of Poly(varepsilon-Caprolactone) (PCL). J. Biomater. Sci. Polym. Ed. 2011, 23, 2197-2206.

35. Putt, K.S.; Pugh, R.B. A high-throughput microtiter plate based method for the determination of peracetic acid and hydrogen peroxide. PLoS ONE 2013, 8, e79218. [CrossRef]

36. Wagner, M.; Brumelis, D.; Gehr, R. Disinfection of wastewater by hydrogen peroxide or peracetic acid: D of procedures for measurement of residual disinfectant and application to a physicochemically treated municipal effluent. Water Environ. Res. 2002, 74, 33-50. [CrossRef] [PubMed]

37. Pruss, A.; Gobel, U.B.; Pauli, G.; Kao, M.; Seibold, M.; Monig, H.J.; Hansen, A.; von Versen, R. Peracetic acid-ethanol treatment of allogeneic avital bone tissue transplants-A reliable sterilization method. Ann. Transpl. 2003, 8, 34-42.

38. Haimi, S.; Vienonen, A.; Hirn, M.; Pelto, M.; Virtanen, V.; Suuronen, R. The effect of chemical cleansing procedures combined with peracetic acid-ethanol sterilization on biomechanical properties of cortical bone. Biologicals 2008, 36, 99-104. [CrossRef] [PubMed]

39. Woon, C.Y.; Pridgen, B.C.; Kraus, A.; Bari, S.; Pham, H.; Chang, J. Optimization of human tendon tissue engineering: Peracetic acid oxidation for enhanced reseeding of acellularized intrasynovial tendon. Plast. Reconstr. Surg. 2011, 127, 1107-1117. [CrossRef] [PubMed]

40. Bokhari, M.; Carnachan, R.J.; Przyborski, S.A.; Cameron, N.R. Emulsion-templated porous polymers as scaffolds for three dimensional cell culture: Effect of synthesis parameters on scaffold formation and homogeneity. J. Mater. Chem. 2007, 17, 4088-4094. [CrossRef] 